\title{
Trauma und Erinnerung - Zur Psychopathologie des autobiographischen Gedächtnisses
}

\author{
Hans-Peter Kapfhammer \\ Univ.-Klinik für Psychiatrie, Medizinische Universität Graz, Graz, Österreich
}

Herstellung und Aufrechterhaltung von personaler und psychosozialer Identität im Fluss der biographischen Veränderungen ist untrennbar mit der menschlichen Kompetenz zu einem autobiografischen Gedächtnis verknüpft. Die Fähigkeit zu einer mentalen Reise sowohl in die persönliche Vergangenheit als auch in eine vorgestellte Zukunft, das hiermit verbundene einzigartige, kohärente und kontinuierliche Selbstgefühl und Selbstbewusstsein kennzeichnet das autobiographische Gedächtnis. In seiner Funktionalität ist es von der Integrität definierter neuropsychologischer und neurobiologischer Strukturen abhängig. Leistungen des autobiografischen Gedächtnisses stellen stets hoch differenzierte Konstruktionen der Persönlichkeit in einer aktuellen Lebenssituation und gegenwärtigen Lebensphase dar. Als motivierte Konstruktionen unterliegen sie auch der Möglichkeit eines Störeinflusses durch mannigfaltige biologische, psychologische und soziale Faktoren. Die enge Verknüpfung von autobiografischem Gedächtnis, Selbst und Identität einer Person stellt somit eine grundlegende psychopathologische Herausforderung dar.

In einem Symposium „Trauma und Erinnerung - zur Psychopathologie des autobiographischen Gedächtnisses" bei der diesjährigen Jahrestagung der ÖGPP in Gmunden wurde dieses Thema aus drei Perspektiven näher beleuchtet.

$\overline{\text { Univ.-Prof. Dr. med. Dr. phil. Hans-Peter }}$ Kapfhammer, Medizinische Universität Graz, Univ.-Klinik für Psychiatrie, Auenbruggerplatz 31, 8036 Graz, Österreich, E-Mail: Hans-peter. kapfhammer@klinikum-graz.at
In einem einführenden Referat (H.P. Kapfhammer) wird der Zusammenhang von Trauma, Dissoziation und posttraumatischer Belastungsstörung aufgenommen. Das klinische Konzept von Dissoziation und dissoziativen Störungen wird dargestellt und an Hand der dissoziativen Amnesie erörtert. In einer ätiopathogenetischen Perspektive wird die allgemeine Beziehung von Trauma und emotionaler Gedächtnisbildung, von Posttraumatischer Belastungsstörung und spezieller Störung des autobiografischen Gedächtnisses dann näher ausgeführt. Die inhärente Spannung zwischen unvollständigem oder gar verlorenem Zugriff auf zentrale Ausschnitte des autobiografischen Gedächtnisses in einer bewussten Erinnerung einerseits, und unwillentlicher intrusiver Erinnerung in der Form desorganisierter und oft nur schwer verbalisierbarer Erinnerungseindrücke andererseits markiert ein häufiges Dilemma von PatientInnen, die nach einer schweren Traumatisierung eine Posttraumatische Belastungsstörung entwickeln. Dieses empirisch ergründbare Dilemma ist auch die Basis für die oft kontroverse Diskussion um die Phänomene von wiederentdeckten (recovered memories) und falschen Erinnerungen (false memories).

Der komplexe Zusammenhang von Erinnerung und Scheinerinnerung in einer posttraumatischen Verarbeitung wird in einem eigenen Beitrag vertieft (T. Stompe). Als zentrales klinisches, aber insbesondere auch herausforderndes forensisch-psychiatrisches Thema müssen Scheinerinnerungen sorgfältig von verwandten Phänomenen wie Irrtum, Selbsttäuschung oder
Lüge abgegrenzt werden. Einen Verständnis fördernden Einblick in dieses oft nur schwierig zu durchdringende Geflecht der subjektiven Erinnerungsfähigkeit können Studienergebnisse der experimentalpsychologischen Gedächtnisforschung verschaffen. Von großem praktischem Nutzen für eine korrekte Erfassung und Beurteilung geschilderter traumatischer Ereignisse sind empirisch erprobte Richtlinien einer gekonnten und sensiblen Exploration.

Auf eine noch schwierigere Ausgangssituation stoßen Kliniker und Gutachter, wenn sie Erinnerungen von Patienten auf ihre Glaubwürdigkeit und Authentizität hin beurteilen müssen, die nicht nur schwerwiegenden psychosozialen Traumatisierungen wie einer Folter ausgesetzt waren, sondern im Rahmen dieser traumatischen Misshandlung auch unterschiedliche Schweregrade eines Schädel-Hirntraumas erlitten haben (K. Papageorgiou). Das Thema der posttraumatischen Amnesie verweist auf den diffizilen Zusammenhang von psychologischer Verarbeitung des Foltertraumas einerseits und hierbei erlittener Hirnschädigung andererseits. Die wichtigsten Ergebnisse aus empirischen Untersuchungen werden skizziert. Die Empfehlungen des Istanbul-Protokolls zum Umgang mit schwer traumatisierten Menschen können helfen, einen unreflektierten Untersuchungsprozess mit dem Risiko einer Retraumatisierung zu vermeiden sowie das Verständnis für das große Dilemma der Glaubwürdigkeit im Erfahrungsbericht eines Folterüberlebenden zu fördern. 